12th International Symposium on Cosmology and

Particle Astrophysics (CosPA 2015)

International Journal of Modern Physics: Conference Series

Vol. 43 (2016) 1660187 (12 pages)

(C) The Author(s)

DOI: $10.1142 / \mathrm{S} 2010194516601873$

\title{
CosPA 2015 and the Standard Model
}

\author{
W.-Y. Pauchy Hwang \\ National Taiwan University, Taipei 106, Taiwan \\ wyhwang@phys.ntu.edu.tw
}

Published 7 July 2016

\begin{abstract}
In this keynote speech, I describe briefly "The Universe", a journal/newsletter launched by APCosPA Organization, and my lifetime research on the Standard Model of particle physics. In this 21st Century, we should declare that we live in the quantum 4-dimensional Minkowski space-time with the force-fields gauge-group structure $S U_{c}(3) \times S U_{L}(2) \times$ $U(1) \times S U_{f}(3)$ built-in from the very beginning. This background can see the lepton world, of atomic sizes, and offers us the eyes to see other things. It also can see the quark world, of the Fermi sizes, and this fact makes this entire world much more interesting.
\end{abstract}

PACS numbers: 12.60.-i, 98.80.Bp

\section{1. "The Universe"}

The International CosPA Symposium has been there, judging from the simple fact that this is the 12th annual International CosPA symposium. But the journal/ newsletter "The Universe" has been on the show for less than three years. Maybe four years ago, I and Ray Volkus chatted about the journal of the organization, the Asia Pacific Organization for Cosmology and Particle Astrophysics (APCosPA Org) and for some reasons we were curious if the journal name "The Universe" has been used before. That was why "The Universe" became the symbol of APCosPA Org.

In Figs. 1-3, we show you the cover of Vol.3 No.1 of "The Universe" (in Fig. 1), the editorial board and others (Fig. 2), and the information which you can retrieve from the high-energy physics network "INSPIRE" (in Fig. 3). Note that we will give out a couple of T.D. Lee prize of "The Universe" to the selected "best" papers published in the journal every three years — see Fig. 2.

Why do we need the journal/newsletter "The Universe" and others from the viewpoint of APCosPA Org? If APCosPA Org could be there for over a hundred

This is an Open Access article published by World Scientific Publishing Company. It is distributed under the terms of the Creative Commons Attribution 4.0 (CC-BY) License. Further distribution of this work is permitted, provided the original work is properly cited. 


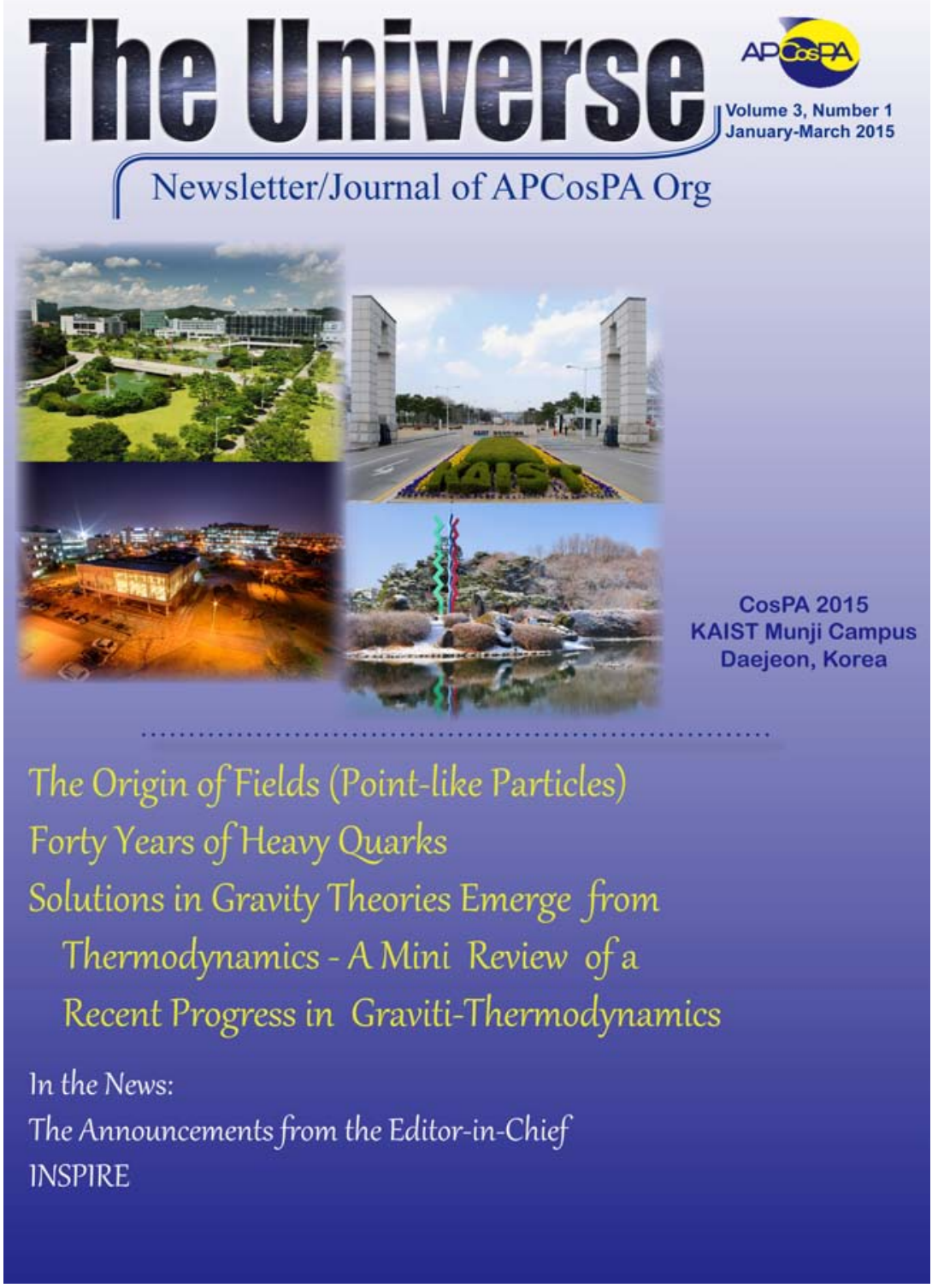

Fig. 1. The cover for The Universe, Vol.3, No.1.

years, then a nice journal such as "The Universe" would be part of the very good memory.

In fact, it would take ten or twenty years to make a very good journal, so beyond one's dream or personal ambition. But if we could safeguard this publication using the standards at a high level, it may eventually become a very good journal, the dreamed "Universe". 


\begin{tabular}{|c|c|c|}
\hline \multicolumn{2}{|c|}{ EDITORIAL BOARD } & \multirow[b]{2}{*}{ ADVISORS } \\
\hline CORE EDITORS & EDITORS & \\
\hline \multirow{3}{*}{$\begin{array}{l}\text { W-Y. Pauchy Hwang } \\
\text { Editor-in-Chief } \\
\text { National Taiwan University, Taipei } \\
\text { Sang Pyo Kim } \\
\text { Kunsan National University, Korea }\end{array}$} & $\begin{array}{l}\text { Paul Ho } \\
\text { Academia Sinica, Taipei }\end{array}$ & $\begin{array}{l}\text { Ernest } M \text {. Henley } \\
\text { University of Washington, Seattle, USA }\end{array}$ \\
\hline & $\begin{array}{l}\text { Pei-Ming Ho } \\
\text { National Taiwan University, Taipei }\end{array}$ & $\begin{array}{l}\text { Bruce H. J. McKellar } \\
\text { University of Melbourne, Australia }\end{array}$ \\
\hline & $\begin{array}{l}\text { Miao Li } \\
\text { Sun Yat-Sen University, Guangzhou }\end{array}$ & $\begin{array}{l}\text { John D. Barrow } \\
\text { University of Cambridge, UK }\end{array}$ \\
\hline $\begin{array}{l}\text { Jen-Chieh Peng } \\
\text { University of Illinois, } \\
\text { Urbana Champagne, USA }\end{array}$ & $\begin{array}{l}\text { Bo-Qiang Ma } \\
\text { Peking University, Beijing }\end{array}$ & $\begin{array}{l}\text { Robert D. Brandenberger } \\
\text { McGill University, Canada }\end{array}$ \\
\hline \multirow{4}{*}{$\begin{array}{l}\text { Bin Wang } \\
\text { Shanghai Jiao Tong University, } \\
\text { Shanghai }\end{array}$} & $\begin{array}{l}\text { Sandip Pakvasa } \\
\text { University of Hawaii, USA }\end{array}$ & $\begin{array}{l}\text { Anthony Zee } \\
\text { ITP, Santa Barbara, USA }\end{array}$ \\
\hline & $\begin{array}{l}\text { Raymond R. Volkas } \\
\text { University of Melbourne, Australia }\end{array}$ & EDITORIALASSISTANTS \\
\hline & $\begin{array}{l}\text { Masahide Yamaguchi } \\
\text { Tokyo Institute of Technology, Japan }\end{array}$ & $\begin{array}{l}\text { Chih-Hsin Huang } \\
\text { Email: chhuang@phys.ntu.edu.tw }\end{array}$ \\
\hline & $\begin{array}{l}\text { Jun'ichi Yokoyama } \\
\text { University of Tokyo, Japan }\end{array}$ & $\begin{array}{l}\text { Szu-Hua Lin } \\
\text { Email: shlin@phys.ntu.edu.tw }\end{array}$ \\
\hline \multicolumn{3}{|l|}{ Manuscript Submissions: } \\
\hline
\end{tabular}

\section{T.D. Lee Prize of "The Universe":}

We would try to select one important paper once every three years - honored as T.D. Lee prize of "The Universe". So, for the first time, one paper from Vol. 1, No.1 to Vol. 3, No.4, 36 papers altogether, are judged from an impartial panel as appointed by the group of the core editors. The prize carries a small amount of cash award (to begin with, US 3,600 ). For the second time and the followings, the pool to be considered would cover 6 volumes altogether, and so on - stopping at previous 9 volumes. The underlying idea is that if the paper is found to be very important even after a few years (at most nine years), it should still be celebrated as T.D. Lee Prize of "The Universe",

\section{The System of the "Core" Editors:}

Each "core" editor can handle, with full authority (equivalent to the Editor-in-Chief), your contributed article, subject to selection of the referees and others. If there is some dispute among the "core" editors, the final decision would be reached by the group of all the "core" editors. Moreover, the new editor-in-chief is selected from one of the core editors when the old editor-in-chief is about to retire at his (her) six-year term.

There are general editors in the pool. Together with the "core" editors, each of them could invite some one for writing the invited paper (which requires at least one referee, recommended by other editor).

Each term of the Editor-in-Chief is for six years; in case of necessary, such term could be renewable. The term of the core editor or of a general editor is for twelve years; or commensurable with two terms of the Editor-in-Chiefs. Unless specified otherwise, the office of the newsletter/journal "The Universe" is located at National Taiwan University, Taipei. The location of the office would not be changed unless something unexpected happened. We insist on such arrangements so that the overall quality would be maintained for many more decades to come.

\footnotetext{
The newsletter/journal "The Universe" is published jointly by the Asia Pacific Organization for Cosmology and Particle Astrophysics and National Taiwan University. (Address: Department of Physics, National Taiwan University, Taipei 10617, TAIWAN).

All rights reserved. This journal or parts thereof may not be reproduced in any form or by any means, electronically or mechanically, including photocopying, recording or any information storage and any retrieval system now known or to be invented, without written permission from the copyright owner.
}

Fig. 2. Continued from Fig. 1, noting about T.D. Lee prize of "The Universe".

In the rest of the "paper", I would like to focus the attention on the so-called "Standard Model of the 21st Century", which we believe it will become one cornerstone of the 21st Century. This is the basic reason which, for so many years, I have been thinking on the final form of the Standard Model, from one corner to the others. Since the year of 2,000 , it is rather disturbing why there are $25 \%$ dark matter while only $5 \%$ visual ordinary matter - a real surprising fact if it is indeed true. 


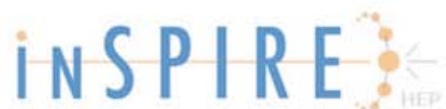

Welcome to INSPIRE, the High Energy Physics information system. Please direct questions. comments or concerns to feedback@inspirehep.net.

\section{Hep $\because$ Hepnames $\because$ Institutions $\because$ Conferences $;:$ Jobs $: \because$ Experiments} JOURNALS $\because:$ HELP

773_p:"Universe" Brief format
findi
"Phys Rev_Lett $105^{* *}$
more
Sort by:
latest first
- or rank by - + Display results:

HEP 23 records found Search took 0.09 seconds.

1. Some studies on dark energies

R-G. Cai (Beiing, Inst. Theor. Phys.), Y. Gong (Beijing, Inst. Theor. Phys. \& Hua-Zhong U. Sci.

Tech.), B. Wang (Beijing. Inst. Theor. Phys. \& Shanghai Jiaotong U., INPAC). 2014. 44 pp.

Published in Universe 2 (2014) 3, 21-64

References | BibTeX | LaTeX(US) | LaTeX(EU) | Harvmac | EndNote Link to Fulltext

Detailed record

2. The curvature perturbation and non-minimal coupling

J. White (Kyoto U., Yukawa Inst., Kyoto). 2014. 19 pp.

Published in Universe 2 (2014) 3, 2-20

References | BibTeX | LaTeX(US) | LaTeX(EU) | Harvmac | EndNote Link to Fulltext

Detailed record

3. Exactly solvable quantum mechanics

Ryu Sasaki (Shinshu U. \& Taiwan, Natl. Taiwan U.). Nov 11, 2014. 48 pp.

Published in Universe 2 (2014) 2, 2-32

e-Print: arXiv: 1411.2703 [math-ph] | PDF

References | BibTeX | LaTeX(US) | LaTeX(EU) | Harvmac | EndNote

ADS Abstract Service; Link to APCosPA server

Detailed record

4. Cosmological perturbations in inflation

Jian-Hua He (Brera Observ.), Bing Wang (Shanghai Jiaotong U.). 2014. 14 pp.

Published in Universe 2 (2014) 2, 33-46

References | BibTeX | LaTeX(US) | LaTeX(EU) | Harvmac | EndNote

Link to APCosPA server; Link to Fulltext

Detailed record

5. The origin of mass

W.Y. Pauchy Hwang (Taiwan, Natl. Taiwan U.). 2014.

Published in Universe 2 (2014) 2, 47-57

References | BibTeX | LaTeX(US) | LaTeX(EU) | Harvmac | End Note Link to APCOSPA

Fig. 3. You download from Inspire, the high-energy physics network. 


\section{Prelude to the Standard Model}

In fact, in 2008 I presented the key part of the idea leading to the Standard Model at Pohang, S. Korea. I conceived the idea that summer in Philadelphia and first presented at CosPA 2008 in Pohang. Over the years, it is getting matured as a whole.

As said earlier, if our Universe turns out to have $25 \%$ dark-matter materials while only $5 \%$ visual ordinary matter, it gives us the biggest puzzle in the natural science. If the unknown $25 \%$ remains to be unknown, then it will be a "joke" in the natural science.

One way out of all these is trying to understand the so-called "Standard Model of particle physics". At least in the Standard Model which I proposed, we do know that the $25 \%$ dark matter must be the neutrinos and antineutrinos, since they are the only long-lived dark-matter particles. The rest of the puzzling question is relatively simple - since neutrinos have (tiny) masses, they will cluster - an important signature of the dark matter.

My efforts in search of the understanding of the $25 \%$ dark matter did begin when I began to be in charge of "the Research Excellence Project on Cosmology and Particle Astrophysics" (2000-2009) under the Ministry of Education of Taiwan and later under National Science Council. The research efforts on the search of the Standard Model are my life-long endeavors, basically.

\section{The Standard Model of the 21st Century}

The late Professor Henry Primakoff, my Ph.D. advisor, spoke at his 60th Birthday Symposium, beginning with the question, "Why is our space three-dimensional? Why our time one-dimensional?" In the late 1970's, I did not know the answer and even did not know this would be a meaningful question. I bet that Henry knew part of the answers except that he did not tell the other people.

Until these years (when I reached 60's and when there is no more pressure for the publications) ${ }^{1}$ I recognized that in fact these are deep meaningful questions. We attempt to describe the point-like particles by the complex scalar fields, the Dirac fields, and the force-fields gauge fields - there are a lot of mysteries (magics) happening with the 4-dimensional Minkowski space-time. In the 4-dimension, the complex scalar field is naturally born with the self-repulsive interaction, $\lambda\left(\phi^{\dagger} \phi\right)^{2}$ with positive $\lambda$ determined by the 4 -dimensional Minkowski space-time (not by the field $\phi$ itself). Thus, the complex scalar field, if alone, would be self-repulsive and cannot exist.

Once we assume that we live in the 4-dimensional Minkowski space-time, the Einstein relation, i.e., $E^{2}=\vec{p}^{2}+m^{2}$, is the origin of everything. The Dirac fields, such as for the electrons, stems from the linearization of the Einstein relations, $E^{2}=\vec{p}^{2}+m^{2}$. The resultant Dirac relation, i.e., $E=\beta m+\vec{\alpha} \cdot \vec{p}$ describes the electron so well. The Dirac algebra, satisfied by those sixteen $4 \times 4$ matrices, is the first place where we may ask why it is 4-dimensional. 
One immediately realizes that if we make the space two-dimensional (and time one-dimensional), the linearization, similar to Dirac's, of the Einstein relation could not fly - it is simply not closed. In this way, we may rule out the dimension from three, from five, etc. The dimension of four, i.e., three spatial dimensions and one for time, is the one selected by the Einstein relation plus the Dirac linearization of the Einstein relation. The algebras, including the Dirac algebra, seem to easily closed at four, but not at three or five. This is the period of our proof.

The Dirac linearization of the Einstein basic relation calls for the two components of the spin, in addition to the existence of the antiparticle. Again, the 4-dimensional Minkowski space-time is the origin of the spin, and also the origin of the antiparticle. Apparently the fundamental importance, in relation to the two-component spin, of the Dirac linearization of the Einstein basic relation was overlooked by physicists throughout the 20th Century. That is, the origin of the spin comes from the requirement of that we have to live in the 4-dimensional Minkowski space-time. All these should enter the textbooks in the 21st Century.

In the 4-dimensional Minkowski space-time, a complex scalar field $\phi(x)$ cannot exist by itself, since the self-repulsive interaction $\lambda\left(\phi^{\dagger} \phi\right)^{2}$ is born with it. This self-repulsive interaction exists because of the 4-dimensional Minkowski space-time, independent of the field $\phi(x)$ itself. This is another magic for the 4-dimensional Minkowski space-time. $\lambda$ is dimensionless (and equals to $\frac{1}{8}$, which is my guess although it seems rather difficult to prove) in this 4-dimensional Minkowski spacetime.

Thus, we live in the (quantum) 4-dimensional Minkowski space-time with the force-fields group $S U_{c}(3) \times S U_{L}(2) \times U(1) \times S U_{f}(3)$ gauge-group structure builtin from the very beginning. ${ }^{2}$ The complex scalar (Higgs) fields now exist in pairs: $\Phi(1,2)$ (SM Higgs), $\Phi(3,2)$ (mixed family Higgs), and $\Phi(3,1)$ (purely family Higgs) jointly to make weak bosons $W^{ \pm}, Z^{0}$ and the family bosons massive. This is the "background" of our world.

In passing, we stress that our description of the "background" of our Universe is evidenced by the presence of the $3^{\circ} \mathrm{K}$ cosmic microwave background (CMB) in our Universe. Because photons are massless, the $3^{\circ} \mathrm{K}$ is rather uniform. On the other hand, the predicted cosmic background (CB) $\nu$ 's, owing to the non-zero mass, would cluster, forming in lumps - this may be the story of the $25 \%$ dark matter (against the only $5 \%$ visual ordinary matter).

The lepton world and, separately, the quark world are accepted by the "background" of our world. ${ }^{2}$ They all come from Dirac's linearization of the Einstein relation $E^{2}=\vec{p}^{2}+m^{2}$. The size of the quark world is typically $(1 \mathrm{fermi})^{3}$, which is so much smaller than that of the lepton world of the atom size $\left(\sim\left(10^{-8} \mathrm{~cm}\right)^{3}\right)$. The Dirac's linearization of Einstein basic relation is needed because the motion of the particle involves the (linear) change in energy.

We may emphasize that the quark world is accepted by the "background" in view of its (123) symmetry (i.e., under $\left.S U_{c}(3) \times S U_{L}(2) \times U(1)\right)$. It is well-behaved when the energy is very large, or $Q^{2} \rightarrow \infty$, or when the distance between two 
quarks is very small, or $r \rightarrow 0$. We propose that the lepton world is protected by another (123) symmetry (i.e., under $\left.S U_{L}(2) \times U(1) \times S U_{f}(3)\right)$ - so, well-behaved as $Q^{2} \rightarrow \infty$, or $r \rightarrow 0$.

In fact, "the lepton world" or "the quark world" may be regarded not just as "a physical system" but also as "a mathematical system". The "consistency" of the system is of utmost importance. In the lepton world or in the quark world, the "constituents" are quantum point-like particles (Dirac fields) in the 4-dimensional Minkowski space-time (with the force-fields gauge-group labels).

To be more specific, in the 4-dimensional Minkowski space-time, what is the behavior of a complex scalar field $\phi(x)$ ? A complex scalar field would have the following Lagrangian:

$$
L=-\left(\partial_{\mu} \phi\right)^{\dagger} \partial_{\mu} \phi-\left\{m^{2} \phi^{\dagger} \phi+\lambda\left(\phi^{\dagger} \phi\right)^{2}\right\}
$$

with the last two terms defining the minus of the potential, $-V(x)$.

$\lambda$ has to be positive to stabilize the system. In only the 4-dimensional Minkowski space-time, $\lambda$ is dimensionless - it must be a universal constant independent of the field itself, or, it is a characteristic of the 4-dimensional Minkowski space-time. We suspect that $\lambda=\frac{1}{8}$, although at present we do not know how to prove this basic aspect.

A positive $\lambda$ means that this self-interaction is repulsive, and so this field cannot exist by itself. Note that, when the temperature is high enough (like in the early Universe), the mass term becomes irrelevant (i.e., very small by comparison). In fact, the Standard Model can be written as a virtually dimensionless theory, with all couplings dimensionless except the "ignition" term. This dimensionless theory is also for the early Universe.

Thus, the lagrangian for the single complex scalar field $\phi$ is fixed (is given) except that the mass term could be adjusted, "fixed" in the 4-dimensional Minkowski space-time. The complex scalar field $\phi$ is self-repulsive and cannot exist.

That is why we must introduce the related complex scalar fields $\Phi(3,2)$ (mixed family Higgs) and $\Phi(3,1)$ (purely family Higgs) to lower the energy and to make the entire story. (In the notations, the first number refers to $S U_{f}(3)$ while the second for $S U_{L}(2)$.) This is the story on the origin of mass. ${ }^{3}$

It is just right to have the three Higgs fields, and only the three, $-\Phi(1,2)$ (the Standard-Model Higgs), $\Phi(3,2)$ (mixed family Higgs), and $\Phi(3,1)$ (purely family Higgs) - to make the family gauge bosons all massive and to make the proper room for neutrino oscillations.

\section{Separation from the Background: The Lepton World and the Quark World}

In the Standard Model, ${ }^{4}$ we live in the quantum 4-dimensional Minkowski spacetime with the force-field gauge-group structure $S U_{c}(3) \times S U_{L}(2) \times U(1) \times S U_{f}(3)$ built-in from the very beginning. This is what we call "the background". 
In this background, the $3^{\circ} \mathrm{K}$ cosmic microwave background (CMB) is there. In view of that photons are massless, their distribution is rather uniform. On the other hand, the anticipated cosmic background $\nu$ 's tend to cluster, in lumps, since we now firmly believe that neutrinos have some tiny masses.

Then, what is the quark world? The quark world is a world of matter form, thus of the type of Dirac equations. It claims a rather small length scale, of about $10^{-13} \mathrm{~cm}$. The strong-interaction nature of $S U_{c}(3)$ explains such small size. The color $S U_{c}(3)$ gauge fields are already classified as part of "the background" - the quarks of three colors and of six flavors are building blocks of matter for the quark world. The quark world knows the gauge group $S U_{c}(3) \times S U_{L}(2) \times U(1)$, but not $S U_{f}(3)$ — the so-called (123) symmetry.

The quark world knows color $S U_{c}(3)$ well - the strong interaction that acts in the range of fermi's (i.e., $10^{-13} \mathrm{~cm}$ ). Everything larger than a few fermi would eventually cut off the influence of the strong interaction, unless some special arrangements are given (by the God).

The lepton world is very similar except that the scale is much bigger, at the atomic scale, or $10^{-8} \mathrm{~cm}$. But it does not know the color $S U_{c}(3)$, except indirectly.

In terms of the size consideration, $10^{-13} \mathrm{~cm}$ versus $10^{-8} \mathrm{~cm}$ so much different, the quark world really has nothing to do with the lepton world. Thus, it is an independent question to ask whether the gauge group $S U_{f}(3)$ would see the lepton world, or whether to see the quark world. On this point, we also are curious why the strong-interaction group $S U_{c}(3)$ sees only the quark world, but not the lepton world.

"Our world" is the combination of the background, the quark world, and the lepton world — so, it is quite complicated but in fact all of them are (quantum) point-like particles. Amazingly, each of them could be represented as a branch of mathematics - or, relativistic quantum mechanics and quantum field theory. ${ }^{5}$

The decomposition of the Standard Model could make our thoughts much clearer. Such as: we live in the (quantum) 4-dimensional Minkowsi space-time with the force-fields gauge-group structure $S U_{c}(3) \times S U_{L}(2) \times U(1) \times S U_{f}(3)$ built-in from the outset - as the "background" of our world. This background supports the quark world. For some reason, it also supports the lepton world.

To treat the family concept in a force-fields gauge-group framework, we regard ${ }^{6}$ $\left(\left(\nu_{\tau}, \tau\right)_{L},\left(\nu_{\mu}, \mu\right)_{L},\left(\nu_{e}, e\right)_{L}\right)($ columns $)(\equiv \Psi(3,2))$ as the $S U_{f}(3)$ triplet and $S U_{L}(2)$ doublet. It is essential to complete the (extended) Standard Model ${ }^{4}$ by working out the Higgs dynamics in detail. ${ }^{3}$ It is also essential to realize the role of neutrino oscillations - it is the change of a neutrino in one generation (flavor) into that in another generation; or, we need to have the coupling $i h \bar{\Psi}_{L}(3,2) \times \Psi_{R}(3,1) \cdot \Phi(3,2)$, exactly the coupling introduced by Hwang and Yan. ${ }^{6}$ Then, it is clear ${ }^{4}$ that the mixed family Higgs $\Phi(3,2)$ must be there. The remaining purely family Higgs $\Phi(3,1)$ helps to complete the picture, so that the eight gauge bosons are massive in the $S U_{f}(3)$ family gauge theory. ${ }^{7}$ 
Remember that the story is pretty much fixed if the so-called "gauge-invariant derivative", i.e. $D_{\mu}$ in the kinetic-energy term $-\bar{\Psi} \gamma_{\mu} D_{\mu} \Psi$, is given for a given basic unit of motion. ${ }^{5}$ It seems that this aspect is as fundamental as the Einstein relation, $E^{2}=\vec{p}^{2}+m^{2}$.

We should stress that all the couplings in the quark world or in the lepton world are dimensionless. Thus, it has to do with the 4-dimensional Minkowski space-time; it has nothing to do with the individual fields. Hence, this story is absolutely beautiful - the act of the Einstein relation and of its Dirac's linearization in the quantum 4-dimensional Minkowski space-time. Either the lepton world or the quark world is the necessary attachment of the 4-dimensional Minkowski space-time. It is a beautiful result, indeed!!

The other important development concerns the understanding of "The Origin of Mass". ${ }^{3}$ For that, we realize that, before the spontaneous symmetry breaking (SSB), the Standard Model does not contain any parameter that is pertaining to "mass", but, after the SSB, all particles in the Standard Model acquire the mass terms as it should - a way to explain "the origin of mass". In this way, we sort of tie "the origin of mass" to the effects of the SSB, or the generalized Higgs mechanism.

In fact, that sets the unique stage for the dimensionless interaction $\lambda\left(\phi^{\dagger} \phi\right)^{2}$ in the 4-dimensional Minkowski space-time. We realized that, to begin with, we have the three Higgs fields and, then, the (elusive) purely family Higgs $\Phi(3,1)$ could work the best as the "ignition" channel, even though this deviates from the standard wisdom of using $\Phi(1,2)$ as the "ignition" channel.

Thus, we have to have the various Higgs at our disposal, but not too many in view of the repulsive nature of these scalar fields. In the model, ${ }^{4}$ we have the Standard-Model Higgs $\Phi(1,2)$, the purely family Higgs $\Phi(3,1)$, and the mixed family Higgs $\Phi(3,2)$, with the first label for $S U_{f}(3)$ and the second for $S U_{L}(2)$. We need another triplet $\Phi(3,1)$ since all eight family gauge bosons are massive. ${ }^{7}$

In other words, we believe that we live in the quantum 4-dimensional Minkowski space-time with the force-fields gauge-group structure $S U_{c}(3) \times S U_{L}(2) \times U(1) \times$ $S U_{f}(3)$ built-in from the very beginning. Only in this world (i.e., our world), the complex scalar fields have those "peculiar" characteristics - these characteristics are determined globally by the 4-dimensional Minkowski space-time, not by the fields themselves.

We may write down all the terms for potentials among the three Higgs fields, subject to (1) that they are renormalizable, and (2) that symmetries are only broken spontaneously (i.e., the generalized Higgs mechanism). This is initially what we do in constructing the Standard Model. ${ }^{4}$

In the U-gauge, we choose to have

$$
\begin{aligned}
& \Phi(1,2)=\left(0, \frac{1}{\sqrt{2}}(v+\eta)\right), \quad \Phi^{0}(3,2)=\frac{1}{\sqrt{2}}\left(u_{1}+\eta_{1}^{\prime}, u_{2}+\eta_{2}^{\prime}, u_{3}+\eta_{3}^{\prime}\right), \\
& \Phi(3,1)=\frac{1}{\sqrt{2}}\left(w+\eta^{\prime}, 0,0\right),
\end{aligned}
$$


all in columns. The five components of the complex triplet $\Phi(3,1)$ get absorbed by the $S U_{f}(3)$ family gauge bosons and the neutral part of $\Phi(3,2)$ has three real parts left — together making all eight family gauge bosons massive.

Basically, we may write down a general renormalizable lagrangian, like in our work in constructing the Standard Model. ${ }^{4}$ But, because of three "cooperative" complex scalar Higgs fields, because of a universal $\lambda$, and because of only one "ignition" point, the real lagrangian becomes rather simple. ${ }^{3}$ It seems that $\lambda\left(=\frac{1}{8}\right)$ should obey some sort of non-renormalization theorem, owing to that it is determined completely by the 4-dimensional Minkowski space-time, not by the complex scalar fields themselves.

It is an interesting question to ask why there is only one "ignition" channel, and whether we could do away with this "ignition" channel. When we examine such questions much further, we realize that, without the dimensional "ignition" term, our theory becomes completely dimensionless, all couplings in the background, in the quark world, and in the lepton world - they are determined completely by the 4-dimensional Minkowski space-time, not by its contents. That is why we have the faith in this theory, the so-called "Standard Model".

In short, we begin with ${ }^{3}$

$$
\begin{aligned}
V_{\text {Higgs }}= & \mu_{2}^{2} \Phi^{\dagger}(3,1) \Phi(3,1)+\lambda\left(\Phi^{\dagger}(1,2) \Phi(1,2)+\cos \theta_{P} \Phi^{\dagger}(3,2) \Phi(3,2)\right)^{2} \\
& +\lambda\left(-4 \cos \theta_{P}\right)\left(\Phi^{\dagger}(\overline{3}, 2) \Phi(1,2)\right)\left(\Phi^{\dagger}(1,2) \Phi(3,2)\right)+\lambda\left(\Phi^{\dagger}(3,1) \Phi(3,1)\right. \\
& \left.+\sin \theta_{P} \Phi^{\dagger}(3,2) \Phi(3,2)\right)^{2}+\lambda\left(-4 \sin \theta_{P}\right)\left(\Phi^{\dagger}(\overline{3}, 2) \Phi(3,1)\right)\left(\Phi^{\dagger}(3,1) \Phi(3,2)\right) .
\end{aligned}
$$

These are two prefect squares minus the other extremes, to guarantee the positive definiteness, when the minus $\mu_{2}^{2}$ was left out. $\left(\theta_{P}\right.$ may be referred to as "Pauchy's angle".)

We obtain ${ }^{3,4}$

$$
v^{2}\left(3 \cos ^{2} \theta_{P}-1\right)=\sin \theta_{P} \cos \theta_{P} w^{2}
$$

And the SSB-driven $\eta^{\prime}$ yields

$$
w^{2}\left(1-2 \sin ^{2} \theta_{P}\right)=-\frac{\mu_{2}^{2}}{\lambda}+\left(\sin 2 \theta_{P}-\tan \theta_{P}\right) v^{2} .
$$

These two equations show that it is necessary to have the driving term, since $\mu_{2}^{2}=0$ implies that everything is zero. Also, $\theta=45^{\circ}$ is the (lower) limit.

The mass squared of the SM Higgs $\eta$ is $2 \lambda \cos \theta_{P} u_{i} u_{i}$ (noting the factor of two), as known to be $(125 \mathrm{GeV})^{2}$. The famous $v^{2}$ is the number divided by $2 \lambda$, or $(125 G e V)^{2} /(2 \lambda)$. Using PDG's for $e, \sin ^{2} \theta_{W}$, and the $W$-mass, ${ }^{8}$ we find $v^{2}=255 \mathrm{GeV}$. So, we set $\lambda=\frac{1}{8}$, a simple model indeed.

The mass squared of $\eta^{\prime}$ is $-2\left(\mu_{2}^{2}-\sin \theta_{P} u_{1}^{2}+\sin \theta_{P}\left(u_{2}^{2}+u_{3}^{2}\right)\right)$. The other condensates are $u_{1}^{2}=\cos \theta_{P} v^{2}+\sin \theta_{P} w^{2}$ and $u_{2,3}^{2}=\cos \theta_{P} v^{2}-\sin \theta_{P} w^{2}$ while the mass 
squared of $\eta_{1}^{\prime}$ is $u_{1}^{2} \lambda$, those of $\eta_{2,3}^{\prime}$ be $u_{2,3}^{2} \lambda$. The mixings among $\eta_{i}^{\prime}$ themselves are neglected in the paper.

There is no SSB for the charged Higgs $\Phi^{+}(3,2)$. The mass squared of $\phi_{1}$ is $\lambda\left(\cos \theta_{P} v^{2}-\sin \theta_{P} w^{2}\right)+\frac{\lambda}{2} u_{i} u_{i}$ while $\phi_{2,3}$ be $\lambda\left(\cos \theta_{P} v^{2}+\sin \theta_{P} w^{2}\right)+\frac{\lambda}{2} u_{i} u_{i}$.

A further look of these equations tells that $3 \cos ^{2} \theta_{P}-1>0$ and $2 \sin ^{2} \theta_{P}-1>0$. A narrow range of $\theta_{P}$ is allowed (greater than $45^{\circ}$ while less than $57.4^{\circ}$, which is determined by the group structure). For illustration, let us choose $\cos \theta_{0}=0.6$ and work out the numbers as follows: (Note that $\lambda=\frac{1}{8}$ is used.)

$$
\begin{gathered}
6 w^{2}=v^{2}, \quad-\mu_{2}^{2} / \lambda=0.32 v^{2} ; \\
\eta: \quad m^{2}(\eta)=(125 \mathrm{GeV})^{2}, \quad v^{2}=(250 \mathrm{GeV})^{2} ; \\
\eta^{\prime}: \quad m^{2}\left(\eta^{\prime}\right)=(51.03 \mathrm{GeV})^{2}, \quad w^{2}=v^{2} / 6 ; \\
\eta_{1}^{\prime}: \quad m^{2}\left(\eta_{1}^{\prime}\right)=(107 \mathrm{GeV})^{2}, \quad u_{1}^{2}=0.7333 v^{2} ; \\
\eta_{2,3}^{\prime}: \quad m^{2}\left(\eta_{2,3}^{\prime}\right)=(85.4 \mathrm{GeV})^{2}, \quad u_{2,3}=0.4667 v^{2} ; \\
\phi_{1}: \text { mass }=100.8 \mathrm{GeV} ; \quad \phi_{2,3}: \text { mass }=110.6 \mathrm{GeV} .
\end{gathered}
$$

All numbers appear to be reasonable. Since the new objects need to be accessed in the lepton world, it would be a challenge for our experimental colleagues.

As for the range of validity, $\frac{1}{3} \leq \cos ^{2} \theta_{P} \leq \frac{1}{2}$. The first limit refers to $w^{2}=0$ while the second for $\mu_{2}^{2}=0$.

Before the family Higgs are discovered, we may try to "guess" the various couplings, using our common senses. $\kappa$, the basic coupling of the family gauge bosons would be the most important in this "guess". The electroweak coupling $g$ is 0.6300 while the strong QCD coupling $g_{s}=3.545$ (order of unity); my first guess for $\kappa$ would be about 0.1 (which is rather small). The masses of the family gauge bosons would be estimated by using $\frac{1}{2} \kappa \cdot w$, so slightly less than $10 \mathrm{GeV}$. (In the numerical example with $\cos \theta_{P}=0.6$, we have $6 w^{2}=v^{2}$ or $w=102 \mathrm{GeV}$. This gives $m=5 \mathrm{GeV}$ as the estimate.) So, the range of the family forces, existing in the lepton world, would be 0.04 fermi.

In, ${ }^{3}$ the term that ignites the SSB is chosen to be with $\eta^{\prime}$, the purely family Higgs. This in turn ignites EW SSB and others. It explains the origin of all the masses, in terms of the spontaneous symmetry breaking (SSB). SSB in $\Phi(3,2)$ is driven by $\Phi(3,1)$, while SSB in $\Phi(1,2)$ from the driven SSB by $\Phi(3,2)$, as well. The different, but related and each self-repulsive, complex scalar fields can accomplish so much, to our surprise. And these Higgs are exactly those the gauge fields (i.e., the force-fields) demand.

In Ref. 3, we have the prediction (or, post-prediction) that $m_{S M}(\eta)=v / 2$, a relation satisfied well. Our criterion is that the "ignition" should cover the "entire" Higgs sector.

The "uniqueness" in the determination of the $\lambda$ means that the choice of the potential is unique ${ }^{3}$ and so the Standard Model is unique. The angle $\theta_{P}$ is the only unknown. 


\section{Concluding Remarks}

We realize that the 4-dimensional Minkowski space-time is the origin of the Standard Model. The Einstein basic relation, $E^{2}=\vec{p}^{2}+m^{2}$, and its Dirac's linearization play the role of generating the "background", the lepton world, and the quark world, leading to the Standard Model. ${ }^{4}$ All couplings are dimensionless, a signature that the 4-dimensional Minkowski space-time is at work.

So far our knowledge concerning the Standard Model does not give us any clue regarding the origin of the extremely feeble gravitational force. Einstein might be surprised that his basic relation and Dirac's linearization is the origin of the Standard Model, while leaving the question of the feeble gravitational force completely open.

\section{Episode}

In the summer of 2008, I went back to U. Penn, my alma mater, attending a high energy physics conference, staying at nearby Sheraton, on the first night waking up at two in the early morning and (suddenly) realizing that what I was doing on the strong interactions (regarding whether Higgs mechanism is relevant in QCD) should be "applied" to the family symmetry, resulting in $S U_{f}(3)$. In the early fall of 2008, I presented the idea at the International CosPA Symposium at Pohang, S. Korea. ${ }^{7}$ So, I felt that it is a special honor to be able to present the resultant Standard Model in this speech, again in S. Korea - somewhat ironic to me (though not to the unknowing audience and to the organizers of this CosPA Symposium as well).

\section{References}

1. W.-Y. Pauchy Hwang, The Universe, 3-2, 11 (2015); "Why is our space-time 4dimensional?". The stories about Primakoff were not told during the speech, because of the limited time. I keep them in this written version for our entertainments.

2. W.-Y. Pauchy Hwang, The Universe, 3-1, 3 (2015); "The Origin of Fields (point-like Particles)".

3. W.-Y. Pauchy Hwang, The Universe, 2-2, 34 (2014); "The Origin of Mass".

4. W.-Y. Pauchy Hwang, arXiv:1340.4705v2 [hep-ph] 25 Aug (2015); "The Standard Model".

5. Ta-You Wu and W.-Y. Pauchy Hwang, "Relativistic Quantum Mechanics and Quantum Fields" (World Scientific, 1991), currently being updated into the second edition.

6. W.-Y. Pauchy Hwang and Tung-Mow Yan, The Universe, Vol. 1, No. 1, 5 (2013); arXiv:1212.4944v1 [hep-ph] 20 Dec 2012.

7. W.-Y. Pauchy Hwang, Nucl. Phys. A844, 40c (2010); W.-Y. Pauchy Hwang, International J. Mod. Phys. A24, 3366 (2009); the idea first appeared in hep-ph, arXiv: 0808.2091; talk presented at 2008 CosPA Symposium (Pohang, Korea, October 2008), Intern. J. Mod. Phys. Conf. Series 1, 5 (2011); plenary talk at the 3rd International Meeting on Frontiers of Physics, 12-16 January 2009, Kuala Lumpur, Malaysia, published in American Institute of Physics 978-0-7354-0687-2/09, pp. 25-30 (2009).

8. Particle Data Group, "Review of Particle Physics", J. Phys. G: Nucl. Part. Phys. 37, 1 (2010); and its biennual publications. 